\title{
Karl-Heinz Ladeur Helmut Ridders Konzeption der Meinungs- und Pressefreiheit in der Demokratie
}

\section{Auf dem Weg zu einer politischen Theorie der Kommunikationsfreibeiten}

Im Zentrum von Helmut Ridders wissenschaftlicher Arbeit der soer und 6oer Jahre hat die Auscinandersetzung um eine politisch und verfassungstheoretisch informierte Konzeption der Meinungs- und Pressefreiheit gestanden. Es soll im folgenden gezeigt werden, daß viele der von ihm entwickelten Ideen auch heute noch für eine Bestimmung des verfassungsrechtlichen Standorts der Kommunikationsfreiheiten von Bcdeutung sind. Viele der Forderungen Ridders an eine politisch reflektierte Verknüpfung zwischen Freiheiten und Demokratietheorie werden praktisch in der ncueren Rechtsprechung des Bundesverfassungsgerichts eingelöst. Bei näherem Hinsehen zeigt sich aber, daß dies vielfach nur auf einer - schon von Ridder früher perhorreszierten $^{r}$ - begriffslosen Abwägung zwischen hererogenen "Interessen " basiert, daß abcr das Bundesverfassungsgericht, und ihm folgend die Literatur, kaum dogmatische Konturen entwickelt hat ${ }^{2}$, die es erlauben, die unaufhebbaren, aber ihre Erscheinungsformen verändernden Konflikte um die Kommunikationsfreiheit und ihre Grenzen so zu strukturieren, daß eine theoretische Selbst- und Fremdbeobachtung der Praxis und ihrer Evolution ermöglicht wird?'

So ist das Bundesverfassungsgericht sicher in den letzten Jahren im Ergebnis in seiner Rechtsprechung vor allem zu den Grenzen der Meinungsfreiheit immer liberaler geworden", aber es muß doch bedenklich erscheinen, daß dieser Wandel sich nicht in Grundsatzentscheidungen vollzogen hat, sondern immer noch den gleichen, vor allem von der Abwägung zwischen individuellen und kollektiven Gütern bestimmten Gesichtspunkten folgt. Dies ist nur ein Indiz dafür, daß vor allem die Diskussion um die Schranken der Meinungsfreiheit nicht zu einer dogmatisch befriedigenden, systematischen und rechtstheoretisch haltbaren Bestimmung der Stellung der Meinungsfreiheit und ihrer Schranken im Verfassungssystem geführt hat. Diese Bedenken werden nicht von einem verselbständigten Intcresse an der juristischen Konstruktion gespeist, vielmchr kommt es auch und gerade unter Bedingungen schnellen Wandels der Medieninhalte und der damit verbundenen Konflikte (z. B. die jüngere Auseinandersetzung um das Verhältnis von Meinungsfreiheit und Persönlichkeitsschutz) darauf an, Bcgriffe zu entwickeln, die jenseits des Einzelfalls verallgemeinerungsfähige Unterscheidungen entwickeln, mit denen Verknüpfungen zwischen Entschei-

\footnotetext{
I Vgl. Dic soziale Ordnung des Grundgesetzes, Opladen 1975, S. $76 \mathrm{E}$.

2 Vgl. nur BVerfGE 7, $19^{8}$ (Luth); 43, 130, 139 (v. Firks); BVerfGE 75, 369, 380 (Strauß/Hachfeld); N]W 1994, 2943; 1995,3303 (Soldaten-Urteilc); zu cinem (nicht recht überzeugenden) Versuch, die Rechtsprechung des BVerfG zu systcmatisiercn vgI. D. Grimm, Die Meinungsfreiheic in der Rechisprechung des BVerfG, NJW 1995, 1697 m.w. V.; vgl. jetzt auch U.F.H. Rühl, Tatsachen - Interpretationen - Wertungen, BadenBaden 1998.

3 Vgl. Th. Vesting, Soziale Geltungsanspruche in fragmentierten Offentichkeiten. Zur neucren Diskussion über das Verhältnis von Ehrenschutz und Meinungstreiheit, AoR 122 (1997), 337 ff.: K. H. Ladeur, Meinungsfreiheit, Ehrenschutz und die Veranderung der Offentlichkeit in der Massendemokratie, AfP r 993. Sistif.

4 Vgl. nur die Nachwcise in Fußnote 2.
} 
dungen hergestellt und sich herausbildende Muster der Entscheidungsfindung konstruiert und beobachtet werden können. Sonst öffnet sich die Rechtsprechung mehr oder weniger unbewußt für Zeitströmungen ${ }^{5}$ - dies ist grundsätzlich unvermeidbar, aber dies sollte durch eine systematisch entwickelte Begrifflichkeit beobachtet werden und sich nicht nur in unterschiedlichen Aufwärts- und Abwärtsbewegungen der "Schaukeltheorie" des Bundesverfassungsgerichts niederschlagen.

Ridder hat schon früh mit seiner vielfach diskutierten Abhandlung über "Die Meinungsfreiheit « im Handbuch »Die Grundrechte « ${ }^{6}$ einen theoretisch anspruchsvollen Ansatz zu Theorie und Dogmatik der Meinungsfreiheit formuliert, die die Kommunikationsfreihcit einerscits vor dem Einschrumpfen auf cin unpolitisches Residuum privater Selbstverständigung und andererseits vor der Funktionalisierung für einen das Öffentliche monopolisierenden Staat bewahren wollte. Beide Tendenzen waren in der Rechtsprechung und Literatur der soer Jahre präsent. Die Gcfahren der einen zeigen sich vor allem daran, daß eine von der Thcorie des Öffentlichen »abstrahierende Konzeption « der Meinungs- und Pressefreiheit bei der Bestimmung ihrer Schranken, insbesondere der "allgemeinen Gesetze" dazu tendieren muß, die sogenannte Drittwirkung der Grundrechte in der Privatsphäre wegen der Staatsgerichtetheit des »Abwehrrechts « eher zu leugnen, aber auch im Strafrecht $z$. B. bei den Beleidigungsdelikten die Meinungsfreiheit mit dem engen Gefäß der Wahrnehmung "berechtigter Interessen « ( $\$$ r93 StGB) auszuschöpfen?.

Natürlich können die "berechtigten Interessen « mehr oder weniger weit gefaßt werden, aber darin ist immer schon die Gefahr einer opportunistischen situationsabhängigen Interessenabwägung angelegt. Gerade deshalb hat sich Ridder auch gegen dic kasuistische Rechtsprechung des Bundesverfassungsgerichts zur Meinungs- und Pressefreiheit gewandt, obwohl das Bundesverfassungsgericht sowohl eine (bcschränkte) Drittwirkung als auch die »institutionellen « Elemente der Presse (und des Rundfunks) anerkannt hat ${ }^{8}$. Insbesondere die Bestimmung der Grenzen der Drittwirkung wird aber immer wieder im Rekurs auf den Einzelfall durch »Abwägung " orientiert: Deren Problematik zeigt sich daran, daß je nach Situation das Gewicht der Meinungsfreiheit von einem persönlichen Interesse des Verfassungsbeschwerdeführers (Lüth) aufgrund seines früheren Engagements für die christlichjüdische Verständigung" oder (R.Schmid) ein berechtigtes Interesse am "Gegenschlag" gegenüber einem seinerseits nicht zimperlichen Gegner ("Der Spiegel «) anerkannt wird ${ }^{10}$. In einem späteren Fall hat das Bundesverfassungsgericht dann die Abwägung von Persönlichkeitsrecht und Meinungsfreiheit (beim Verteilen eines Flugblattes) auf die Schriftgröße und den erforderlichen Leseaufwand der Adressaten abgestellt, um zu dem Ergebnis zu kommen, der Verfasser des Flugblattes habe eine aufmerksame Lektüre vorausgesetzt, deshalb dürfe nicht auf den Eindruck eines oberflächlichen Lesers abgestellt werden (v. Firks)" . - Die Reihe ließe sich bis in die Gegenwart fortsetzen'2. Die genauere, nicht oberflächliche, den Umfang der Begründungen des Bundesverfassungsgerichts berücksichtigende Interpretation seiner Urteile wird auch bei der Lektüre im zeitlichen Abstand feststellen, daß eine

s Vgl. zum Wandel der Dogmatik der Meinungsfreiheit in den USA M. A. Graber, Transforming Free Specth, Burkeley etc. 1991

6 H. Ridder, Dic Mcinungsfreiheit, in: Neumann/Bettermann/Scheuner, Die Grundrechte, Bd. 2, Berlin I $9 \$ 4$ (2. Aufl. 1968), S. 242 ff

7 Vgl. dazu krit:sch H. Ridd́er, JZ 1961, 538 (Anm. zum R. Schnid-Beschluß des BVerfG); W. Mallmann, Pressepflichten und moffentliche Aufgabe* der Presse, JZ 1966, 625, $626 \mathrm{tf}$.

8 Vgl. BVerfGL 10, 118, 121; 20, 162, 175f.

9 BVerHE $7,198 \mathrm{ff}$

10 BVerfGI: $12,1_{13}=\mathrm{JZ} 1961,535 \mathrm{~m}$. Anm. Ridder.

II BVerfGI: $43,130,139$.

12 BVerfG, NJW 1994, 2943; 1995, 3303 (Soldaten). 
Vielzahl von Gesichtspunkten gegeneinander abgewogen werden und man sich deshalb leicht ausmalen kann, daß das Urteil bei einer geringfügig anderen Wortwahl oder anderen Umständen auch ganz anders hätte ausfallen können. Diese Neigung des Bundesverfassungsgerichts - auch in guter Absicht und häufig mit glücklichem Ausgang -, Abwägungen unter einer Vielzahl von Interessen und Gesichtspunkten vorzunehmen, bietet jedenfalls keine tragfähigc Grundlage für eine theoretisch reflektierte Dogmatik der Kommunikationsfreiheit's.

Die andere Variante der Verfehlung der Eigenständigkeit der Grundrechte auf Meinungs- und Pressefreiheit besteht für Ridder in der Anerkennung der politischöffentlichen Funktion der Grundrechte bei gleichzeitiger Funktionalisicrung für den Staat als Träger öffentlicher Entscheidungsgewalt. Die Basis für solche Theorien ist schon in der Weimarer Republik gelegt worden, sie sind aber auch in den soer Jahren weiterentwickelt worden. Ein Beispiel dafür bietet R. Smend, der für (vor allem) politische Grundrechte wie die Meinungs- und Pressefreiheit auch ihre Leistung als »Verstärkungen des Staates und der Staatsgewalt « betrachtet. Die Grundrechte haben danach "mindestens ihrer Intention nach einen Teil der Funktionen des monarchischen Verfassungselements übernommen«(!), nämlich die Symbolisierung von Einheit zu gewährleisten. Dic Konsequenzen dicser Verstaatlichung des Öffentlichen schlagen sich auch im Verständnis der »allgemeinen Gesetze «als Schranke der Grundrechte der Meinungs- und Pressefreiheit nieder: Als »allgemein « gelten danach »diejenigen Gemeinschaftswerte, die als solche den ursprünglich individualistisch gedachten Grundrechtsbeschränkungen gegenüber den Vorrang haben, so daß ihre Vertretung eine Überschreitung, ein Mißbrauch des Grundrechts ist «'t. Die damit verbundenen Gefahren liegen auf der Hand. Aber auch die Grenzen der privatistischen Konzeption der Abwehrrechte kommen in den Schwierigkeiten bei der Bestimmung des »allgemeinen Gesetzes « zum Ausdruck, denn sie muß ein Konzept von der Bedeutung der Konfliktfähigkeit der Meinungsfrciheit mit diesen Gesetzen entwickeln, um das Verhältnis zwischen privatem und öffentlichem Interesse zu konkretisieren's.

Dieser Schwierigkeit haben sich verschiedene Ansätze zur Interpretation des Konzepts der »allgemeinen Gesetze " gestellt. Eine eher formale, vor allem in den USA verbreitete Unterscheidung von Handeln und Meinung (als Nicht-Handeln) kann durchaus an liberale Traditionen anknüpfen und einen theoretisch reflektierten Ansatz formulieren, auch wenn sie im Einzelfall ihre grundsätzliche Unterscheidung nicht durchhalten kann ${ }^{16}$. Das Bundesverfassungsgericht hat die begrifflichen Unterschiede zwischen den verschiedenen Konstruktionen zur Bestimmung des "allgemeinen Gesetzes « eingeebnet und eine Formel gefunden, die formale und materielle Interpretation trotz ihres Gegensatzes miteinander verbindet und im einzelnen auf die Abwägung bei der Anwendung des Gesetzes vertraut, die es vorher großzügig als "allgemein" klassifiziert hat. Es gibt danach so gut wie keine allgemeinen Gesetze mehr, es kommt nur noch darauf an, bei der Interpretation den besonderen Wert der Meinungsfreiheit zu berücksichtigen. H. Ridder hat dies später als den Versuch der Ersetzung rechtswissenschafrlicher Konstruktion und rechtspraktischer Dogmatik durch Orientierung an "Wirklichkeiten « bezeichnet ${ }^{17}$.

Wenn man die Rechtsprechung exemplarisch analysiert, wird man dem durchaus

13 Vgl. abcr Grimm (Fn. 2).

14 Vgl. R. Smend, Staatsrechrliche Abhandlungen, 2. Aufl., Berlin 1968, S. $92 \mathrm{ff}$.

is Vgl. nur F. Forsthoff, Tagespresse und Grundgesetz, DÖV $1963,633 \mathrm{ff}$.

$16 \mathrm{Vgl}$. Graber (Fn. 5), S. 184; G. Gounalakis, Freiraume und Grenzen politischer Karikatur und Sacire, NJW i99s, sogff.

17 Vgl. Ridder (Fn. r), S. 46 
zustimmen können. So fällt vor allem in den letzten Jahren auf, daß die Rechtsprechung scharfe Töne im Meinungskampf nicht im Hinblick auf eine bestimmte Konzeption der Öffentlichkeit akzeptiert, sondern weil man sich tatsächlich aufgrund der "Reizüberflutung " nicht mehr anders Gehör verschaften kann, also weil sich bestimmte Formen der Äußerung faktisch verbraucht haben ${ }^{18}$.

Einc weitere Konfliktlinie zwischen Pressefreiheit und Gesetzgebcr hat sich später vor allem in der Auseinandersetzung um solche Gesetze entwickelt, die in anderer Weise als durch Ziehung materieller Schranken für die Verbreitung von Meinungsinhalten in den Pressebereich intervenieren: Ein Beispiel dafür bietet die Diskussion über die gesetzliche Einführung sogenannter innerer Pressefreiheit ${ }^{59}$. Die in den 6oer Jahren aus der Redakteursbewegung hervorgegangene Idee, dic zu verschiedenen Gesetzesentwürfen geführt hat, paßt nicht in die begrifflichen Schcmatisierungen der älteren Konzeptionen. Besonders dieser Konflikt wird von Ridder immer wieder zur Demonstration seiner eigenen These herangezogen, daß die Pressefreiheit einer eigenständigen Sphäre des Öffentlichen zugerechnet werden muß, die weder mit der Privatsphäre noch mit dem Staat identifiziert wcrden kann ${ }^{20}$. Die Problematik läßt sich daran zeigen, daß ein Gesetz über die Mitbestimmung der Redakteure nur dann als ein Eingriff in die Pressefreiheit verstanden werden kann, wenn man diese mit der Presseunternehmerfreiheit der Verleger identifiziert. Andercrseits muß im Angesicht zunehmender Pressekonzentration, die nicht allein durch dic Instrumente des GWB bewältigt werden kann, die Möglichkeit einer Selbstgefährdung der Pressefreiheit durch dic Presse akzeptiert werden. Die Vertreter einer individualistischen Konzeption der Pressefreiheit wollten dies, da es sich hier nicht um einen "Eingriff « im engeren Sinne handeln kann ${ }^{21}$, als einc Art »systemimmanenter Einschränkung des Grundrechts " durch das Demokrarieprinzip konstruieren". Die widersprüchliche Formel läßt immerhin das zu bewältigende, aber schwer zu lösende Problem erkennen.

Der Rückblick auf die rechtsdogmatische Literatur der soer und 6ocr Jahre mag auch deshalb aufschlußreich sein, weil die Kontroverse durchaus dogmatisch und theoretisch reflektierte Unterscheidungen erkennen läßt, die in der gut geölten Abwägungsmechanik des Bundesverfassungsgerichts abgeschliffen werden. Vor diesem Hintergrund muß man dic auch bei Ridder selbst nicht fehlenden dogmatischen Konstruktionsprobleme, auf die noch zurückzukommen ist, in einem anderen Licht sehen: Sie legen Konflikte offen, die vielleicht im einzelnen nur Behelfslösungen zulassen, abcr allein schon dadurch einc aufklärerische Wirkung haben, daß sie nicht vorschncll durch Einzelfallentscheidungen beseitigt werden.

\section{Die Diskussion um die "institutionelle Komponente" der Pressefreiheit}

Die Konstruktionsprobleme der Grundrechtslehren der soer und 6oer Jahre sind nicht zuletzt auch damit zu erklären, daß der Staat sich immer mehr durch die Gesetzgebung einerseits, aber auch durch die Integration der Gruppen in das Verfahren der Erzeugung rechtlich bindender Entscheidungen andererseits einem Funk-

$18 \mathrm{Vgl}$. nur BGH, NJW r994, 124 (Hoechst/C:reenpeace); BVcrfGE GI, 13.

19 Vgl. H. Ridder, Probleme der inneren Pressefreiheit. Der Journalist (Bcilage zum Mai-Heft) 1962

20 Vgl. Ridder (I'n. G), S. 257; ders., Dic offericliche Aufgabe der Presse im System des modernen Verfassungsrechts, Wien: Verband Osterreichischer Zeitungsherausgeber, 1962

21 Vgl. Maun\%-Durig, Art. s Anm. 184 (Stund 1970).

22 Vgl. E. Schwark, der Begriff der "Allgemeinen Geserze* in Art. s Abs. 2 GG, Berlin 1970, S. 106. 
tionswandel unterzieht und sich innerhalb eines neuen Systems des Staat und Gesellschaft verschränkenden sozialen und kulturellen Pluralismus neuer Rechtsformen bedient, die nicht mehr auf das klassische Instrumentarium des "Eingriffs « in Freiheit und Eigentum festgelegt sind. P. Häberle ${ }^{23}$ hat in seiner Dissertation die Wesensgehaltsgarantie der Grundrechte in Art. I9 Abs. 2 GG als den Sitz einer umfassenden Befugnis des Staates angesehen, grundrechtlich gewährleistete Handlungsfelder jenseits der durch Abwehrrechte gegen staatliche Intervention zu verteidigenden Grenzen zxvischen privatem und öffentlichem Interesse erst »einzurichten «. Diese höchst ambivalente Konzeption hat sich in ihrem begrifflichen Ansatz nicht durchgesetzt. Der Sache nach hat sic aber eine Entwicklung auf den Begriff gebracht, die sich in einer dogmatisch weniger artikulierten Form (auch bei Häberle selbst) nicht zuletzt in der Anerkennung "objektiv-rechtlicher « Gehalte der Grundrechte niedergeschlagen hat, die das Bundesverfassungsgericht dann durch seine Abwägungsformeln gegen die subjektiv-rechtlichen Komponenten der Abwehrrechte zum Ausgleich bringt $\mathrm{t}^{24}$.

Auch H. Ridder hat die Notwendigkeit gesehen, die Grundrechte nicht nur auf Abwehrrechte im klassischen Sinne festzulegen, weil damit im Bereich der Kommunikationsfreiheiten die Gefahr verbunden ist, "das Öffentliche" ganz im Staat zu lokalisieren und dic Frciheitsrechte auf die Abwehr von Eingriffen in die Privatsphäre zu begrenzen. Dies ist ein gerade für die Meinungsfreiheit problematischer Ansatz. Eine Auffangposition für die Entwicklung einer eigenständigen Rolle des politischen Gehalts der Meinungsfreiheit läßt sich dann nur im Rekurs auf die erwähnte, in den USA verbreitete Unterscheidung von "Handlung « und "Meinung « aufbauen, mit der Konsequenz, daß der Staat sich aus dem Bereich des Meinens herauszuhalten hat und erst bei der Überschreitung der Grenze zum Handeln intervenieren darf ${ }^{25}$. Daß auch diese Grenze nicht unproblematisch ist, weil die Abgrenzung nur auf den ersten Blick begrifflich durchzuhalten ist, praktisch aber eine Reihe schwieriger dogmatischer Probleme aufwirft ("offensive speech «, "words that hurt «), spricht nicht gegen diesen Ansatz. Aber diese liberale, stark individualistische Konzeption der Mcinungsfreiheit, die H. Ridder in seinem berühmten Aufsatz über die Meinungsfreiheit im Ansatz auch selbst übernommen hat, kann allein der Öffentlichkeit als einer zwischen der Privatheit und der organisierten Staatlichkeit situierten Sphäre der 》Osmose " von Staat und Gesellschaft, ihrer wechselseitigen Durchdringung bei gleichzeitiger rechtlicher Unterscheidbarkeit, nicht gerecht werden ${ }^{26}$. Um der Unterscheidbarkeit dieser »dritten Ebene" der Vermittlung zwischen Privatsphäre und staatlichem Willen kann es auch nicht einfach um die Erstreckung des aut den Staat bezogenen, auf Einheit der Entscheidung angelegten Demokratiegebotes auf die Gesellschaft gehen ${ }^{27}$, und zwar weder auf die Privatrechtsgesellschaft noch auf die Sphäre der Öffentlichkeit selbst. Dies ist vor allem deshalb problematisch, weil damit wieder hinterrücks die an der Einheit des Staates und der Entscheidung orientierten Zwänge in Anschlag gebracht werden können, die sich z. B. auch mit der durchaus wohlmeinend in den Pressegesetzen formulierten "öffentlichen Aufgabe « der Pressc verbinden lassen: Nicht die Presse hat eine öffentliche Aufgabe, sondern die Pressefreibeit ${ }^{28}$. Damit ist eine wichtige Sinnverschicbung verbunden. An die Presse wird keine öffentliche Aufgabe

23 Die Wesensgehaltsgarantie des Art. 19 Abs. 2 GG. Zugleich ein Bertrag zum institutionellen Verstandnis der Grundrechte und der Lehre vom Gesetzesvorbehalt, Karlsruhe 1962.

24 Vgl. nur BVerfGE. $49,89, x_{4} 1 \mathrm{f}$.

25 Vgl. Graber (Fn. 5), S. 148,18 s

26 Vgl. Ridder (Fn. 6), S. $254 \mathrm{ff}$.

27 Vgl. H. Ridder, Die Stellung der Gewerkschaften im Sozialstaat nach dem Grundgesetz fur die Bundesrepublik Deutschland, Sruttgart 1960, S. i iff.

28 Vgl. Ridder (Fn. 1), S. 81 ff.; ders. (Fn. 6), S. 258; Mallmann (Fn. 7) 
"delegiert« - deshalb hat Ridder sich auch kritisch gegenüber dem Verständnis der Presse als einer »vierten Gewalt« geäußert -, sondern in der auf Selbstdefinition durch die Prcsse angelegten Freiheit ist die besondere Aufgabe begründe ${ }^{29}$. Deshalb ist auch die Vorstellung von der "Vermittlung" des Öffentlichen zwischen dem Privaten und dem Staatlichen nur mit Vorsicht zu übernehmen. Die Öffentlichkeit ist nicht auf die Vor-Strukturierung der organisierten (staatlichen) Öffentlichkeit angelegt. Damit wäre eine Unterbewertung der Selbstdefinition der Öffentlichkeit, ihrer Generierung der Themen und Formen öffentlicher Meinungsbildung, aber auch dcr notwendigen Infrastruktur (Sammlung und Speicherung von Informationen, Organisation der Presscunternehmen etc.), der Wahl der Formen der Meinungsäußerung (sachliche Analyse, Satire etc.) verbunden.

Auch hier hat das Bundesverfassungsgericht einige Schritte zur Entwicklung einer institutionellen Konzeption der Presse- (und Rundfunk-) Freiheit getan. Im "Spiegel $\ll$-Urteil hat das Gericht die Notwendigkeit des Schurzes der informationellen Infrastruktur der Presse (Archiv, Informantennetz etc.) auch jenseits der materiellen Freiheit zur Äußerung von Meinungen als dem Schutzbereich von Art. 5 Abs. I S. 2 $\mathrm{GG}$ zugehörig angesehen ${ }^{3 \circ}$. An anderer Stelle hat das Bundesverfassungsgericht auch die Rolle der Parteien nach Art. 2s GG als von der Vor-Strukturierung der Meinungsbildung durch Entwicklung entscheidungsfähiger Positionen (im Hinblick auf die Regierungsbildung) bestimmt ${ }^{3}$. Dic Kehrseite dieser Betrachtungsweise läßt sich einmal an der mangelnden dogmatischen und theoretischen Verknüpfung zwischen »insticutionellen " und subjektiv-rechtlichen Komponenten der Pressefreiheit und insbesondere ihren Folgen in der Konstruktion der Parteien als janusköpfiger "Verfassungsorgane "beobachten: Auf der einen Scite sind die Parteien zum Organstreitverfahren zugelassen, auf der anderen Seite legitimiert die Effizienz der Regierungsbildung die Zulassung der $5 \%$-Klausel im Interesse der Bildung "funktionsfähiger " Mehrheiten im Parlament ${ }^{32}$.

Die Einordnung der Presse und der Parteien als "Institutionen", die jenseits der Sphäre privater Intercssenverfolgung auf die Erhaltung und Gewährleistung öffentlicher Kommunikation angelegt sind, wird von Ridder aufgenommen und im Interesse der Sicherung der Autonomie, insbesondere der Presse, dogmatisch und konstruktiv entfaltet. Mangels einer Alternative bedient er sich dabei des Konzepts der »Institution $\alpha^{33}$, um den prozeßhaften, sich nach selbst erzeugten Regeln organisierenden Bereich des "Öffentlichon" zum Ausdruck zu bringen ${ }^{34}$. Die Übernahme dieses Begriffs hat Ridder später selbst als "unglückselig" bezeichnet, obwohl der Gebrauch des Konzepts eigentlich selbst nicht mißverständlich war ${ }^{35}$. Er war immerhin durch die Assoziationen besetzt, die mit Carl Schmitts Begriffsbildung verbunden waren ${ }^{36}$ : Die institutionellen und Institursgarantien sind nach Carl Schmitt als ein objektiv-rechtlicher Bestand von Normen zu verstehen, die - obwohl nicht einem Grundrechtsbereich zuzuordnen - doch verfassungsrechtlich mindestens partiell gegen Veränderung durch den Gesetzgeber geschützt sind (das gilt etwa danach für die öffentlich-rechrliche Institution des Beamtentums nach Art. 33 Abs. 5 GG und für die privatrechtliche Institutionsgarantic des Eigentums nach Art. $14 \mathrm{Abs}$. I $G G$ ), und dies führt Carl Schmitt darauf zurück, daß bestimmte "Einrichtungen« nur

29 Vgl. H. Ridder, in: E. Heinitz/ders., Landesverrat und Pressefreiheit, Bonn: ASJ, 1965.

30 Vgl. BVerfGE 20, 162, 176.

$31 \mathrm{Vgl}$. BVerfGE 20,56, rooff

$32 \mathrm{Vgl}$. BVerfGE 1, 208, $256 \mathrm{ft}$

33 Vgl. Ridder (Fn. 6), S. 257.

$34 \mathrm{Vg}$. auch Ridder (Fn. 20), S. I r.

3) V l. auch H. Ridder, DVBI r 963,741 (Rezension Dagroglou).

36 Vgl. C. Schmitt. Freihcitsrechte und inscitutionelle Garantien der Reichsverfassung, Leipzig 1931. 
dadurch gesichert werden können, daß ein Bestand von Regeln der Disposition des einfachen Gesetzgebers entzogen wird. Die Verwendung des Begriffs der "Institution « durch H. Ridder war ganz anders gemeint, hat aber vermeidbare Mißverständnisse ausgelöst. Ihm ging es darum, mit diesem Begriff die Eigenständigkeit des politisch fungierenden Grundrechts der Pressefreiheit auch gegenüber der individuellen Meinungsfreiheit zu konstruieren. Eine gewisse Ähnlichkeit zu der Begriffsbildung bei Carl Schmitt besteht aber darin, daß es auch bei H. Ridder um die Sicherung eines Prozesses der Selbstdefinition von Regeln der Öffentlichkeit geht, allerdings mit dem bedeutsamen Unterschicd, daß es sich nicht um staatlich gesetztes Recht, sondern vielmehr um die selbstorganisierten Regeln der öffentlichen Meinungsbildung handelt, die durch dic Presse selbst generiert werden und nicht im individuellen Ausdruck von Meinungen aufgehen.

Für Helmut Ridder sind Meinungs- und Pressefreiheit nicht nur als zwei verschiedene, nebeneinander stehende Grundrechte zu betrachten - dies entspricht durchaus auch der Auffassung des Bundesverfassungsgerichts -, sondern es besteht ein grundsätzlicher Unterschied zwischen der individuellen Meinungsfreiheit und der minstitutionellen « Pressefreiheit ${ }^{37}$ : Meinungsfreiheit ist letztlich der Sphäre des Privaten zuzuordnen (was nicht heißt, daß sie nicht ihrerseits des Schutzes gegen staatliche Funktionalisierung in der Gestalt von Abwehrrechten bedarf); die Pressefreiheit, die den gesamten Prozeß der Informationssammlung und -verarbeitung einschließlich der verschiedenen Funktionen des Redakteurs, Verlegers, aber auch des Leserbriefschreibers, des Grossisten ctc. umfaßt, erfordert aber eine andere Form der rechtlichen Sicherung. Mit dem Konzept der "Institution" war gerade die Garantie der offenen dynamischen Selbstdefinition der politischen Presse als Medium ihrer Professionalisicrung verbunden. Damit sollte die unpolitische Presse nicht generell abqualifiziert werden, weil gerade die Garantie der Selbstdefinition der Presse auch eine staatliche Fremddefinition der Grenzen des Politischen verbieten wür$\mathrm{de}^{38}$, aber damit sollte akzentuiert werden, daß der Schutz der Presse um des politischen Selbstverständnisses der Öffentlichkeit zwischen Privatem und Staatlichem verfassungsrechtlich zu gewährleisten sei.

H. Ridder ist in seinem berühmten Aufsatz über die "Meinungsfreiheit" sogar so weit gegangen, die politische Presse ganz aus dem Schutzbereich von Art. s Abs. I S. 2 GG herauszuverlagern und sie Art. 2x GG als der Grundnorm des vergesellschafteten Staates zuzuordnen ${ }^{39}$, der kcin Monopol auf die Definition des öffentlichen Intcresses in Anspruch nehmen kann und sich im Gegenteil auch jenseits der rechtlich institutionalisierten Kanäle (Wahl, Öffentlichkeitserfordernisse für parlamentarische Verfahren etc.) für die kritische Öffentlichkeit durchlässig halten muß und nur im Hinblick auf das Entscheiden rechtliche Bindung erzeugen kann, nicht aber für die Definition der öffentlichen Interessen selbst. Aus dieser Verknüpfung von Presseund Parteienfreiheit folgt, daß auch die Presse an der politischen Willensbildung des Volkcs »mitwirkt« - bei gleichzeitiger »institutioneller « Unterscheidung der Prcsse als Forum der Öffentlichkeit.

Zugleich ergibt sich daraus wiederum die Bindung der Presse an ihre eigene Funktion der Ver-Öffentlichung von Interessen zwischen der Privatrechtsgesellschaft und der staatlich organisierten Öffentlichkeit: In der pressebezogenen Lesart von Art. 2 r Abs. I S. 3 und 4 GG folgt daraus ein Verfassungsgebot der demokratischen Ordnung der Presse und ihrer Pflicht zur Offenlegung von Abhängigkeiten. Dęmokratie in

37 Vgl. Ridder (Fn. 6), S. 257

$38 \mathrm{Vgl}$. Ridder (Fn. 20), S. 21.

39 Vgl. Rudder (Fn. 20), S. 1s; ders. (Fn. 6), S. 257. 
diesem Sinne ist nicht als Zwang zur Angleichung an die für den staatlichen Bereich bestehenden Demokratiegebote zu verstehen, d. h. es geht nicht um das Erfordernis der "Delegation « von Funktionen durch Wahlen, vielmehr gcht es um dic Sicherung der Selbstdefinition der Presse im Interesse der Demokratie, das Demokratieprinzip verlangt danach unterschiedliche Lesarten für die staatlich verfaßte Öffentlichkeit und die gesellschaftlich aggregierte Sphärc des Öffentlichen.

Diese kühne Konstruktion hat $\mathrm{H}$. Ridder so später nicht wieder aufgenommen, sie geht auch wohl über die Möglichkeiten der grammatikalischen und systematischen Interpretation des Grundgesctzes hinaus, an der er sich sonst zu orientieren versucht, wenn er z. B. die Besonderheit der Pressefreiheit schon darin im Wortlaut des Grundgesetzes selbst angedeutet sieht, daß sie nicht mit der Person verbunden ist. Ob diese Konstruktion für die Konkretisierung eines dogmatisch und theoretisch informierten Verständnisses der Kommunikationsfreiheiten geeignet ist, erscheint im übrigen zweifelhaft. In späteren Arbciten ist Ridder die Norwendigkeit deutlich geworden, den Abstand von Kommunikations- und Parteienfreiheit dogmatisch aufrecht zu erhalten.

Deshalb hat er in seiner "Sozialen Ordnung des Grundgesetzes", die im Jahre I97s erschienen ist, eine andere Konstruktion entwickelt ${ }^{4^{\circ}}$ : Hier wird die Verknüpfung von Presse- und Parteienfreiheit nicht wieder aufgenommen, statt dessen spricht er hier, sowohl die Krücke der "Institution « als auch die Anlehnung der dogmatischen Konstruktion an Art. 21 GG hinter sich lassend, von der Notwendigkeit, das Grundrecht der Pressefreiheit als ein »inpersonales « zu qualifizieren (darauf ist weiter unten noch einmal zurückzukommen).

Art. 2I Abs. 2 GG behält aber auch für die Presse eine wichtige Funktion: Die Möglichkeit des Parteiverbots und die damit verbundenen, über den Bereich der Parteien hinausreichenden staatlichen Eingriffe in die Öffentlichkeit sind dadurch verfassungsrechtlich begrenzt, daß sie nur auf der Grundlage einer Entscheidung des Bundesverfassungsgerichts erfolgen dürfen. Im Umkehrschluß ergibt sich daraus für Ridder die Notwendigkeit, die besonderen strafrechtlichen Sanktionen zum Schutz der "freiheitlichen demokratischen Grundordnung", die im Zeichen des Kalten Krieges eingeführt worden sind, für verfassungswidrig, wcil kompetenzwidrig zu crklären $^{41}$. Nur die Partei als Organisation, nicht aber Ideen dürfen aus dem Prozeß der öffentlichen Meinungsbildung ausgeschlossen werden.

Dadurch wird Ridders Grundkonzeption des Schutzes einer eigenständigen Sphäre des Öffentlichen als Verfahren der Selbstdefinition konsequenterweise auch verfahrensrechtlich abgestützt. Daraus ergibt sich eine Stärkung der Konfliktfähigkeit des Öffentlichen gegenüber der institutionalisierten Staatlichkeit. Das Öffentliche und das Staatliche stehen nicht in einem harmonischen, auf "Integration " angelegten Verhältnis, dessen Bestimmung im einzelnen der staatlichen Entscheidung (Gesetzgeber, Verwaltung und Rechtsprechung) obliegt. Soweit es um den Prozeß der Meinungsbildung selbst geht, bedarf es vielmehr besonderer "Kollisionsregeln«, die dann auch vom Bundesverfassungsgericht interpretiert und konkretisiert werden können, aber nur sehr begrenzt der gesetzlichen und administrativen Einschränkung unterliegen. Prinzipiell muß nämlich der Konflikt zwischen einer Meinung (insbesondere in der Presse) und dem staatlichen Interesse ausgehalten werden, er kann vom Verfahren der Aberkennung der Grundrechte in Art. 18 GG abgesehen ${ }^{42 / 43}$ nicht durch staatliche Entscheidung ausgeräumt werden. Nur soweit es um die

40 Vgl. Ridder (Fn. I), S. 8 s ff.

$4^{1}$ Vgl. Ridder, Grundgesetz, Notstand und politusches Strafrecht, Frankfurt/M. 1965; ders. (Fn. 1), S. 69 f. $4^{2 / 43}$ Vgl. auch H. Ridder/E. Steın, Die Fretheir der Wissenschaft und der Schutz von Staatsgeheımnissen, DOV $1962,361 \mathrm{ff}$. 
Gefährdung von Rechtsgütern durch Handlungen geht, darf das staatlichc Recht der

Meinungs- und Pressefreiheit Schranken ziehen und sie durch Entscheidung konkretisieren. Diese verfahrensrechtliche Dimension der Kommunikationsfreiheit, die Kommunikation als Verfahren selbst entspricht, wird auch durch Ridders Interpretation des Zensurverbots bestätigt: Nach herrschender Meinung handelt es sich dabei nicht um ein Grundrecht, sondcrn eine sogenannte Schrankenschranke $42^{44}$, d. h. auch eine sonst nach Art. s Abs. 2 GG in der Form eines "allgemeinen Gesetzes « zulässige Beschränkung der Meinungsfreiheit ist soweit unzulässig, als damit durch Auferlegung eines Genehmigungserfordernisses die Veröffentlichung begrenzt werden soll. Demgegenüber ist Ridder der Auffassung, daß das Zensurverbot durchaus eine eigenständige grundrechtliche Komponente hat, nämlich den bcsonderen Schutz der Vor-Publikationsphase einer Äußerung besonders gegen den administrativen Zugriff, und zwar nicht nur in der Gestalt der Auferlegung von Genehmigungserfordernissen. Damit ist eine Erweiterung des Anwendungsbereichs des Zensurverbots verbunden, das nach der herrschenden Auffassung praktisch kcine Bedeutung mehr hat: Für Ridder ist damit auch der Schutz der Zugänglichkeit von Informationen zu bestimmten staatlich kontrollierten Teilräumen des Öffentlichen (Schulen, Kasernen etc.) vor Zensur verbunden.

Auch diese Auffassung ist ebensowenig wie die im folgenden darzustellende Konzeption des "allgemeinen Gesetzes" ohne Probleme, aber auch hier hat sie den Vorteil, bestimmte Grenzen der begrifflichen Einordnung offenzulegen und sich der Problembereinigung zu entziehen, die sich einer nicht mehr zeitgernäßen Fixierung auf vergangene Formen der Zensur bedient. Ridder geht es dagegen darum, die Kollision zweier Paradigmen zu institutionalisieren, nämlich den Konflikt zwischen der auf Einheit angelegten Sphäre der Staatlichkeit und der auf Erhaltung und Schaffung von Konflikten um Meinungen angelegten Öffentlichkeit zu ermöglichen und nicht durch Vorrangentscheidungen zugunsten des Staatlichen aufzulösen.

Der oben erwähnte inpersonale Charakter der Pressefreihcit wird bei Ridder leider nicht weiter ausgearbeitet, deshalb mag die Figur die Frage aufwerfen, so what?, wenn dann doch ein Typus von Gesetzen eingeführt wird, das Ausgestaltungsgesetz, das nicht die inhaltliche Pressefreiheit beschränkt, sondern sie durch (staatliches!) Gesetz ermöglicht und entfaltet's. Diese Ausgestaltungsgesetze hat auch das Bundesverfassungsgericht insbesondere in seiner Rechtsprechung zur Rundfunkfreiheit als einen besonderen Regelungstypus weiterentwickelt: Das Ausgestaltungsgesetz schützt, vereinfacht gesagt, nicht die Kommunikationsfreiheit im Interesse eines anderen, nicht der Kommunikation zuzuordnenden Rechtsguts, sondern ermöglicht und beschränkt Konflikte innerhalb der Prcsse selbst, indem es die Reflexrechte der an der Presse beteiligten Subjekte ncu ordnet (deshalb ist ein Gesetz, das die innere Pressefreiheit stärken soll, kein »Schrankengesetz" und folglich auch nicht am Maßstab der "Allgemeinheit « im Sinne von Art. s Abs. 2 GG zu messen). Das Bundesverfassungsgericht prüft solche Gesetze insbesondere auf ihre Geeignetheir im Hinblick auf die damit erfolgende Fremdregulierung von Selbstregulierung. Dies hat zu Einwendungen geführt, daß Kommunikationsfreiheir damit nur nach Maßgabe der Gesetze bestehe.

$44 \mathrm{Vgl.} \mathrm{Jarass/Pieroth,} \mathrm{GG,} \mathrm{4.} \mathrm{Aull.,} \mathrm{München} \mathrm{1998,} \mathrm{Art.,} \mathrm{Rdnr.} 52$.

45 Vgl. BVerfGE 20, 162, 176 (Presse); 57, 295, 320 (Rundfunk). 
Dies ist in der Tat eine Paradoxie, die sich nicht ohne weiteres auflösen läßt! Dennoch erscheint die Konstruktion durchaus fruchtbar: Der inpersonale Charakter öffentlicher Kommunikationsfreiheiten besteht vor allem darin, daß nicht von vornherein bestimmte individuelle Rechtspositionen in Bezug genommen werden, sondern dynamische Erfordernisse der öffentlichen Meinungsbildung postuliert werden, die ganz und gar nicht mit dem quasi-räumlichen Bild eines geschützten Freiheitsbereichs des Individuums übcrcinstimmen. Diese Konstruktion ist durchaus ambivalent, deshalb sind die Einwände gegen eine "Freiheit nach Maßgabe des Gesetzes" nicht von vornherein von der Hand zu weisen. Auf der anderen Seite ist aber zu berücksichtigen, daß (nicht nur) die Presse als Prozeß durch Selbstorganisation zwangsläufig einen distribuierten, an die Koordination unterschiedlicher Positionen gebundenen Charakter annimmt. Daran knüpft die Ausgestaltungsgesetzgebung an. Sie hat damit zugleich einen kooperativen Charakter, der sich aus dem Zwang zur Beobachtung und Evaluation der professionellen und ökonomischen Sclbstorganisation der Presse ergibt ${ }^{4}$, die sich durch Evolution herausgebildet hat. Daß die subjektiv-rechtlichen Positionen eher als "Reflexrechte « qualifiziert werden, bedeutet nicht, daß der Staat sie nach seiner eigenen Rationalität modellieren dürfte. Nur soweit sich die Presseentwicklung ihrerseits gegen Vielfaltstandards wendet, also ihren offenen prozeßhaften Charakter selbst zu zerstören beginnt, ist eine Ausgestaltung nötig. Daneben geht es um die rechtliche Sicherung der Infrastruktur (Organisation und Verfahren) der Presse, die ohnehin nur mittelbar einer staatlichen Funktionalisierung zugänglich sind. Der Staat hat also nur eine Kompetenz zur prozeduralen, grundrechtsgerechten Reorganisation von Kommunikationsfaktoren, die weder die Inhalte noch die Vielfalt der Presse (und des Rundfunks) tangieren darf. Auch dies ist eine durchaus ambivalente Position, der man sich aber nicht dadurch entziehen kann, daß man die Entwicklung der Presse (und des Rundfunks) ganz der Disposition der gegenwärtigen Träger von Reflexrechten überläßt.

Wenn man den Zwang zur Anknüpfung an die Selbstorganisation der Presse selbst und die kooperative Verschränkung von Perspektiven akzentuiert, erweist sich diese wechselseitige Beziehung zwischen Sclbstorganisation und Selbstregulierung der Presse sowie ausgestaltender Fremdregulierung als ein durchaus produktiver Gedanke. Es geht dann verfassungsrechtlich um die Formulierung einer Art Kollisionsrecht, das Fremdregulicrung nur so weit zuläßt, wie Selbstorganisationsprozesse in der Presse sich selbst zu blockieren drohen oder - etwa im Rundfunk, jedenfalls in der Vergangenheit - Freiheit nur durch eine staatliche Vorgabe für die Organisation von Vielfalt möglich erschien. Jedenfalls erweist sich die Formel von der inpersonalen Freiheit insofern als produktiv, als sie im Gegensatz zur Unterstellung einer objektivrechtlichen Dimension der Grundrechte die Selbstorganisation von Regeln durch einen geschützten Handlungsbereich stärker akzentuiert und damit "Ausgestaltung " von vornherein auf die Kooperation von Selbst- und Fremdregulierung festlegt. Inpersonale Freiheit bezeichnet damit den Schutz der Selbstdefinition eines Prozesses der Meinungsbildung, dem auch die Fähigkeit zur Reflexion auf seine Regelhaftigkeit zugeschrieben wird. Diese Fähigkeit zur Selbstorganisation wird durchaus plausibel als Freiheit geschützt, deren inpersonaler Charakter sich darin zeigt, daß es nicht um die Selbstdefinition von Individuen, sondern die distribuierte Erzeugung einer eigenständigen Regelhaftigkeit geht ${ }^{47}$.

46 Vgl. dazu allgemein Th. Vesting, Prozedurale Rundfunktreiheit, Baden-Baden 1997.

47 Interessanterweise hat G. Teubner das Konzcpt des sinpersonalen « Rechts für die Gewährleistung der 
IV. Die "Allgemeinen Gesetze" im Sinne von Art.s Abs. 2 GG und die Selbstorganisation der Öffentlichkeit

Das Interesse an der Erhaltung der Konfliktfähigkeit des Öffentlichen schlägt sich auch in Helmut Ridders Auffassung zur Interpretation der "allgemeinen Gesetze« im Sinne von Art. 5 Abs. 2 GG nieder: Dazu hat er bekanntlich im Anschluß an Häntzschels in der Weimarer Zeit vertretene Position die These begründet, "allgemeine Gcsetze « im Sinne dieser Vorschrift seien solche, die nicht die »rein geistige Wirkung “ einer Meinung beschränkten ${ }^{48}$. Das Bundesverfassungsgericht hat - wie oben erwähnt - die damit im einzelnen verbundenen Interpretationsprobleme dadurch bewältigt, daß es eine glättende Abwägungsformel eingeführt hat. Damit sind alle Unterscheidungen letztlich eingeebnet und durch einzelfallbezogene $A b$ wägungen von Interessen ersetzt worden.

In Ridders Konzeption geht es - um im Bild des Kollisionsrechts für die Abstimmung von Regeln der Selbstdefinition des Öffentlichen im Medium der Presse (und des Rundfunks) und der staatlichen Fremdregulierung zu bleiben - um eine Art "renvoi «"⿱ A : Auf der ersten Stufe läßt sich durchaus mit der einfachen Formel des Schutzes der "geistigen Wirkung « der Meinungen operieren, wenn man auf die typischen Wirkungen eines verhaltenssteuernden Gesetzes abstellt. Andererseits gibt es eine Vielzahl von Gesetzen, die gravierende Nebenwirkungen erzeugen können, etwa Steuergesetze, die Sonderlagen im Bereich der Presse nicht berücksichtigen. Angesichts der komplexen Regelungsanforderungen wäre es nicht akzeptabel, ein allgemeines Gesetz sozusagen nur an seinem eigenen Anspruch, an seinen Absichten zu messen und gravierende Nebenwirkungen außer Betracht zu lassen. Aus dem prozeßh haften Charakter der auf Selbstdefinition angelegten Sphäre der Öfentlichkeit läßt sich dann in umgekehrter Perspektive die Forderung ableiten, daß typische Effekte auf die Selbstorganisation der Presse die "Allgemeinheit« eines Gesetzes ausschließen können. D. h. die Definition darf nicht nur aus der Sicht des Staates erfolgen, sondern ist auf eine kooperative Perspektivenverschränkung zwischen staatlicher Regelung und Selbstregulierung der Öffentlichkeit angelegt. Ein Gesetz muß danach in einem ersten Schritt an seinem eigenen Anspruch zur Fremdregulierung gemessen werden, d. h. es muß danach gefragt werden, ob das Gesetz beabsichtigt, typischerweise die „geistige Wirkung « der Kommunikation zu beeinträchtigen. Sodann muß in einem zweiten Schritt gefragt werden, ob das Gesetz entgegen seinem expliziten $Z$ weck aus der Sicht der Presse eine typische Nebenwirkung erzeugt, die sich als Hindernis für die "geistige Wirkung « der Presse erweist. Auch dann kann das Gesetz nicht als »allgemein « angesehen werden.

Ein besonderes Problem ist mit dem Vorbehalt des gesetzlichen Schutzes der Jugend und der persönlichen Ehre nach Art. 5 Abs. 2 GG verbunden. Nach der herrschenden Meinung hat diese Hervorhebung neben den sallgemeinen Gesetzen « cigentlich keine Bedeurung, da sie diesen Begriff so weit faßt, daß daneben für weitere Vorbehalte im Grunde kein Raum mehr ist. Für Ridder ist aber die "bcsondere* Ausweisung dieser Rechtsgüter, die Beschränkungen der Meinungsfreiheit rechtfertigen, deshalb signifikant, weil es sich bei ihnen eben gerade typischerweise um Gesetze

Eigenstandigkeit von Diskursen stark gemacht ("Diskursrechte 4 ) - und zwar unabhangig von Ridders Begriffsbildung; vgl. Vertragsweiten. Das Recht in der Fragmentierung von Private Governance Regimes, RHJ 1998, 234, 258; vgl. auch ders./Ch. B. Graber, Art and Money, Constitutional Rights in the Private Sphere?, Oxford Journal of Legal Srudies 1998, 6 Iff.

$48 \mathrm{Vgl.} \mathrm{Ridder} \mathrm{(Fn.} \mathrm{6),} \mathrm{S.} 282$.

49 Auch hier bietet sich eine Parallele zu G. Teubner an (The King's Many Bodies: The Self-Deconstruction of the Law's Hierarchy, Law and Sociery Review 1997, 76 ff.). 
handelt, die die Meinungs- und Pressefreiheit beschränken. Ridder sieht darin, nicht ohne Konsequenz, eine Bestätigung seiner formalen Interpretation des »allgemeinen Gesetzes«. Das kann nicht bedeuten, daß der Schutz der Öffentlichkeit hier eine offene Flanke hat und erst im Einzelfall entschieden werden kann, ob nach Abwägung der Schutz der Meinungsfreiheit oder der Schutz der persönlichen Ehre den Vorrang erhält. Vielmehr muß hier - so wäre an Ridder anzuknüpfen - nicht einfach abgewogen werden, sondern es ist genauer danach zu fragen, ob der Konflikt nicht, obwohl es hier nicht allein auf die "geistige Wirkung " der Meinung ankommen kann (anders als im Fall des "allgemeinen Gesetzes"), gerade weil es um diese "geistigen Wirkungen" geht, primär an die Öffentlichkeit selbst zurückgegeben werden kann. Was das bedeuter, läßt sich etwa am Beispiel des Konflikts zwischen Presse-/Kunstfreiheit und Persönlichkeitsschutz im Falle »Mephisto « demonstrieren $^{s e}$ : Hier hätte man davon ausgehen können, daß der Streit durchaus produktiv in der Öffentlichkeit selbst ausgetragen werden kann, weil es durchaus auch um die öffentlichen Resonanzen auf die Selbstdarstellung Gustav Gründgens' in der Öffentlichkeit und ihre Infragestellung durch den Roman von Klaus Mann geht. Es ließe sich die These aufstellen, daß solange und soweit ein solcher Streit produktiv in der Öffentlichkeit selbst ausgetragen werden kann, eine gerichtliche Entscheidung allein deshalb nicht zulässig ist.

In dicsem Fall kommt es ebenfalls zu dem als "renvoi « bezeichneten Effekt: Obwohl die persönliche Ehre grundsätzlich eine Schranke der Meinungsfreiheit bildet, muß hier gefragt werden, ob die Kollision zwischen "geistigen Gütern" selbst, dem Recht auf Meinungsäußerung und dem Recht auf persönliche Ehres", ihren "Ausgleich * durch einen Effekt der Selbstorganisation innerhalb der Öffentlichkeit finden kann, oder ob dies wieder an den Staat (hier das Gericht) zur Entscheidung zurückgegeben werden muß. Letzteres ist vor allem dann der Fall, wenn es um die Veröffentlichung von Daten aus der Privat- oder Intimsphäre geht, oder um Personen, an deren Interessen die Öffentlichkeit nicht in der Form eines Pro und Kontra Anteil nimmt. Diese Auffassung ist sicher differenzierungsbedürftig; dabei läßt sich aber vor allem wiederum konsequenterweise an Ausdifferenzierungen unterschiedlicher Typen von Publikationen denken, die auch zu einer Fragmentierung der Öffentlichkeit geführt haben. $\mathrm{Zu}$ unterscheiden wäre etwa zwischen der allgemeinen Presse, satirischen Organen, Plakaten, Fernsehshows, Versammlungen etc. ${ }^{\text {22 }}$. Hier ließe sich in Kooperation zwischen staatlicher Fremdregulierung und der Selbstdefinition der Medien ein Regclwerk herausbilden, das bestimmte Formen der Bewältigung des Konflikts $z$ wischen Öffentlichkeit und persönlicher Ehre festlegen könnte. Dies ließe sich an einem weiteren Beispiel demonstrieren: Das Bundesverfassungsgericht hat einen in der Literaturzeitschrift "Der Rabe veröffentlichten Verriß von Werken Heinrich Bölls durch den Schriftsteller E. Henscheid als nicht mehr vom Recht der Meinungsfreiheit gedeckt angeschen's. Hier hätte man z. B. darauf abstellen können, daß gerade in einer Literaturzeitschrift der Schutz des geschmähten Autors durch die Entwicklung von Gegenpositionen ausreichend gewährleistet ist. Der Beschluß des Bundesverfassungsgerichts ist gleichzeitig ein gutes Beispiel für die Willkürlichkeit einer kasuistischen Rechtsprechung, die auf der einen Seite primitivste Formen einer

so BVerfGE 30, 173 ff.; vgl. allg. H. Ridder, Die Freiheit der Kunst nach dem Grundgesetz, München 1963. s1 V Vl. dxzu Ridder, JZ 1961, 539, wo die Unterscheidung zwischen allgemeinen und besonderen Gesetzen (zum Schutz) der Ehre entfaltet wird: Auch dic Einfügung einer besonderen - d. h. gegen Meinungsinhalte gerichteten - Grundrechesschranke legitimert weder zu einer umstandslosen Anwendung z. B. des cinschlagigen Strafrechts noch zu begriffsloser Abwagung; auch hier bedarf es einer regelhaften $\Lambda$ bstimmung vun Meinungsfreiheit und E.hrenschutz.

s2 Vgl. Vesting (Fn. 46).

s3 BVerfG, NJW 1993, 1462 (Nicheannahmebeschluß). 
Fäkalsprache als "Satire" nobilitiert" ${ }^{\text {t4 }}$, auf der anderen Seite aber für den Hintersinn der groben Sprache eines literarischen Verrisses keinen Sinn hat und sich offenbar auch nicht näher gefragt hat, warum wohl eine angesehene Literaturzeitschrift eine Schmähschrift gegen Böll veröffentlicht hat.

Es sollte wenigstens deutlich geworden sein, daß die hier-im Anschluß an H. Ridder - vertretene Position gerade nicht auf eine Einzelfallabwägung hinausläuft, sondern sich auch im Fall des Konflikts zwischen »Kommunikationswerten " um die Formulierung eines Kollisionsrechts bemüht, das rechtliche Interventionen in den Prozeß der Meinungsbildung auf die Kooperation von Selbst- und Fremdbeobachtung der Regeln öffentlicher Kommunikation verweist.

Auch wenn $\mathrm{H}$. Ridder mit scinen verfassungsrechtlichen Konstruktionen experimentiert hat und sie dogmatisch nicht immer im Detail ausgearbeitet hat, so hat er gerade durch seinen theoretischen Scharfsinn eine Leistung erbracht, an die theoretisch wieder angeknüpft werden kann - zumal die Schwächen der Rechtsprechung des Bundesverfassungsgerichts zur Meinungsfreiheit nicht allcin durch cine andere Abwägung im Detail zu korrigieren sind. Gerade die dynamische Selbstveränderung der Öffentlichkeit und die Ausdifferenzierung unterschiedlicher Kommunikationsformen macht die Suche nach einer theoretisch reflekticrten dogmatischen Konstruktion des Status der Kommunikationsfreiheiten im Verfassungssystem um so wichtiger.

Ridders Konzeption der Kommunikationsfreiheit soll abschließend an einem weiteren Testfall demonstriert werden, nämlich der Frage nach der Drittwirkung der Grundrechte, insbesondere im Bereich der Meinungsfreiheit selbst. Das Bundesverfassungsgericht hat bekanntlich seine Konzeption dazu insbesondere im LüthUrtcil zu den Schranken der Meinungsfreiheit entwickelt und sie in einer Vielzahl von Einzelfällen weiter differenziertss: Für Ridder ist auch dies eine unbefriedigende Scheinlösung; die Eigenständigkeit der Öffentlichkeit muß sich auch hier darin zeigen, daß, so wenig wie der Staat seine Geltungsansprüchc gegenüber der öffentlichen Kommunikation ohne Kontrolle am Maßstab der Selbstdefinition der Öffentlichkcit selbst durchsetzen kann, auch die Privatsphäre und die darin angelegten Zwänge (Eigentum, Arbeitsvertrag, Schutz von eigentumsähnlichen Rechten etc.) der öffentlichen Kommunikation Schranken setzen darf - außer durch allgemeine Gesetze (und das Recht der persönlichen Ehre). Auch hier zeigt sich, daß eine begrifflich differenzierte Position zu einer verfassungstheoretischen Selbstaufklärung der Dogmatik beitragen kann und eine begriffslos bleibende "Schaukeltheorie" des Bundesverfassungsgerichts keine Orientierung bieten kann. Die Dogmatik kann sich an den Gesichtspunkten orientieren, die schon oben für die Koordination zwischen schrankenziehenden allgemeinen Gesetzen und der Selbstregulierung der Öffentlichkcit genannt worden sind. Auch im Hinblick auf privatrechtliche Auseinandersetzungen gilt, daß die Grenzen des Öffentlichen nur in Kooperation zwischen der Selbstregulierung der Kommunikation einerseits und in dicsem Fall der Selbstregulierung etwa der Privatrechtsgesellschaft andererseits gefunden werden können ${ }^{56}$. Soweit es dabei um Erfordernisse der Kommunikation gcht, die durch die Evolution der Öffentlichkeit selbst formuliert, erprobt und bewertet worden sind, erhält die Öffentlichkeit grundsätzlich den Vorrang. Das bedeutet aber keineswegs, daß stets das Interesse der Meinungsbildung sich durchsetzen wird: Z. B. ist durchaus nicht einzusehen, daß es einem Erfordernis der öffentlichen Meinungsbildung entspricht, daß 
man das Eigentum des Vermieters einer Wohnung für politische Propaganda in Anspruch nehmen kann'?

\section{Resümee}

Die Skizze sollte deutlich gemacht haben, daß Helmut Ridders Beitrag zu einer Dogmatik der Kommunikationsfreiheiten nicht nur retrospektiv zum Verständnis der Rechtsgeschichte der Bundesrepublik von Bedeutung ist, sondern daß daran auch heute angeknüpft werden könnte (und sollte). Ich vermute, daß einige der hier versuchten Weiterentwicklungen selbst nicht Ridders Beifall finden werden. Aber diese Überlegungen könnten insoweit den minpersonalen « Charakter auch der Meinungsbildung über Rechtsdogmatik demonstrieren, die sich Anregungen verdankt, die über das hinausgehen, was der Anregende selbst gemeint hat.

Rainer Ahlers

Doping und strafrechtliche Verantwortlichkeit

Zum strafrechtlichen Schutz des Sportlers vor Körperschäden durch Doping

2., unveränderte Auflage

Doping ist in allen Bereichen des Sports weit verbreitet. Die Sportverbände können es mit verhältnismäßigem Aufwand jedoch allenfalls im Spitzensport reduzieren. Ob das geltende Strafrecht ein wirksames Mittel zu seiner Bekämpfung is sucht R. Ahlers. Zugleich gibter dem Rechtsanwender, der die Strafbarkeit wegen eines Körperverletzungs- und Tölungsdelikts prüft, wertvolle Hinweise zur Lösung seiner sowohl materiell- als auch beweisrechtlichen Probleme. Dabei wird deutlich, daß es vor allem die oftmals vernachlïssigten praktischen Besonderheiten des Dopings sind, die theoretisch anwendbare Vorschriften leerlaufen lassen.

1998. 240 S., brosch., 79,- DM, 577.- öS. 72,- sFr, ISBN 3-7890-5553-0

(Nomos Universitätsschriften - Recht / Unterreihe: Kicler Schriften zum Strafrecht, Bd. 10)

NOMOS Verlagsgesellschaft

76520 Baden-Baden

57 Vgl. BVerfGE 7, 231 ff.; kritssch dazu aber Ridder (Fn. I), S. 71 f. 